\title{
A cross sectional pilot study to estimate the prevalence of and risk factors for leptospirosis in South-Western Victorian dairy herds, 2017
}

\author{
E Erregger**, MA Stevenson, D Beggs, S Oswin, SP Jagoe, PD Mansell and M Pyman \\ *Melbourne Veterinary School, University of Melbourne, 250 Princes Highway Werribee
} 3030

**e.erregger@student.unimelb.edu.au

\begin{abstract}
Leptospirosis is a zoonosis, found worldwide, affecting many species of animals. We conducted a cross-sectional study to estimate the prevalence of Leptospira borgpetersenii sv Hardjo and Leptospira interrogans sv Pomona in cattle in dairy herds in South-Western Victoria, Australia. Fiftythree herds were enrolled into the study. Urine samples were collected from 15 late-lactation cows in each herd. A questionnaire was provided to herd managers at the time of each herd visit, asking them to describe the methods they used for controlling leptospirosis, including vaccination. Urine samples were pooled at the herd level and tested for leptospira spp. using real time PCR. Urine samples from individual cows within the positive pooled samples were then tested for Leptospira Hardjo and Leptospira Pomona using qPCR.
\end{abstract}

Four of the 53 herds showed positive leptospirosis results giving an apparent prevalence of 8 (95\% CI 1 to 13) leptospira-positive herds per 100 herds at risk. Based on the 53 completed questionnaires, leptospirosis vaccination programs were not compliant with label directions in 35 of the 52 vaccinated

This is the author manuscript accepted for publication and has undergone full peer review but has not been through the copyediting, typesetting, pagination and proofreading process, which may lead to differences between this version and the Version of Record. Please cite this article as doi: $10.1111 /$ avj.12984

This article is protected by copyright. All rights reserved. 
herds: 67 (95\% CI 54 to 78) of 100 herd managers that routinely vaccinated for leptospirosis did not comply with label directions. One herd was completely unvaccinated.

Based on our findings, we estimate that approximately $10 \%$ of dairy farms in South-Western Victoria are likely to be infected with leptospirosis. While most herds are vaccinating for leptospirosis, most are not doing so according to label directions. We conclude that herd managers need to be better educated regarding leptospirosis vaccination programs.

\section{Introduction}

Leptospirosis has been identified in most domestic and wild mammalian species ${ }^{1}$. Studies of Leptospira borgpetersenii sv Hardjo (L. Hardjo) and L. interrogans sv Pomona (L. Pomona) carried out in the 1980s in Victoria, Australia, demonstrated a relatively high prevalence of exposure in both cattle (40\% for L. Hardjo and 2\% for L. Pomona ${ }^{2}$ ) and humans $\left(22 \%{ }^{3}\right)$. These estimates need to be revisited as leptospirosis vaccination programs are now commonplace in Victorian dairy herds. If vaccination programs are working well, a decline in herds with cows shedding the bacterium would be expected, as would the risk for leptospirosis in humans ${ }^{45}$.

The size of dairy herds in Australia is increasing and there is a trend away from familyowned farms toward corporate style farms ${ }^{6,7}$. In 2017-2018, approximately 37\% of farms were expecting to increase their herd size in the following year ${ }^{8}$. Simultaneously, the diversity of farming staff has increased. In Australia the use of foreign workers, backpackers 
and holidaymakers is common, which brings people onto farms that might not have been exposed to Leptospira before ${ }^{9}$.

Australian law requires farm staff to be provided with a safe work environment. People working with dairy cattle, especially those working in milking sheds are at increased risk of exposure to leptospirosis and subsequent zoonotic disease ${ }^{10}$, especially if cows in the herd are unvaccinated or vaccinated ineffectively. It is common for cows to urinate during the milking process, especially when being stressed and/or exposed to new people. Direct or indirect contamination of the mucus membranes (e.g. eyes, oral mucus membranes) or broken skin by drops or aerosols of infected urine may be enough to cause infection in humans.

Although leptospirosis is a notifiable disease in all Australian states, relatively little data has been published about the disease in Australian cattle. A possible reason for a lack of reporting the disease is the relatively low pathogenicity of L. Hardjo in cattle in Australia.

In the early 1970s in Australia leptospirosis was often associated with signs of clinical mastitis. The first Victorian case study ${ }^{11}$ reported udders that appeared flaccid, had no swelling or heat, with abnormal milk in all quarters. These infections were associated with L. Hardjo. A study investigating causes of abortion in cattle in Victoria from the late $1980 \mathrm{~s}^{12}$ identified a weak association between abortions and leptospira, despite a high seroprevalence in cattle (28\% for Hardjo and $12 \%$ for Pomona), suggesting a high proportion of cattle being exposed to the bacteria with no clinical effect. 
The disease was not only seen in Victorian cattle. Serovar Pomona was reported as a cause of calf leptospirosis in 1951 in Western Australia ${ }^{13}$, and an investigation into the reproductive performance of beef cattle in Central Queensland describes L. Hardjo and Pomona ${ }^{14}$ in cattle grazed on high and low water containing soils.

Most of these data are outdated. More recent research has been reported from New Zealand, where the pathogen is a serious zoonotic risk to people working in the livestock industry ${ }^{15}$. In New Zealand another strain, sv Tarassovi, has emerged ${ }^{16}$, which is not covered by vaccines on the market.

Compared to the low pathogenicity in Australia, overseas studies, especially in the United Kingdom, have shown a correlation between leptospira seropositivity of cows and reproductive performance ${ }^{17,18}$.

In the UK, in addition to L. borgpetersenii sv Hardjo, L. interrogans serovar Hardjo subtype Hardjoprajitno is also present ${ }^{19}$. Studies of Australian isolates have only identified $L$. borgpetersenii serovar $\operatorname{Hardjo}^{20}$.

The nomenclature for leptospira has changed over time and older articles often use the nomenclature of $L$. interrogans serovar Hardjo subtype Hardjobovis but differentiate between Hardjobovis and $\mathrm{H}$ 
ardjoprajitno. Before 1989 there were only two species of Leptospira reported: L. interrogans, containing all pathogenic leptospira strains, and L. biflexa, containing all saprophytic strains ${ }^{21}$. Since 1989 the phenotypic classification has been replaced by the genotypic classification, and both pathogenic and saprophytic strains now can be found within a single species. The nomenclature contains more genome species, and some serovars are found in more than one species, like sv Hardjo. Serovar Hardjo can be found in species L. borgpetersenii, which would be the only serovar found worldwide including Australia, and species L. interrogans, which mainly is found in the United Kingdom.

In humans leptospirosis is often misdiagnosed because the clinical presentation can be nonspecific and may be mistaken for other causes of acute febrile illness ${ }^{22}$. In patients with risk factors for leptospirosis, a high index of clinical suspicion is important to ensure prompt diagnosis. Given the non-specific signs of disease it is likely that leptospirosis in human populations in Australia is under-reported. Whilst vaccines for leptospirosis in humans exist in other countries, they contain serovar specific antigens and protect only against homologous or closely related, but not heterologous, serovars ${ }^{23}$. Human vaccines available are based on L. interrogans ss Icterohemorrhagiae ${ }^{24}$, L.interrogans serogroup Canicola sv Canicola, Icterohemorrhagiae sv Copenhageni and Pomona sv Mozdok ${ }^{25}$. There are no leptospiral vaccines available for use in humans in Australia. 
Studies indicate that livestock workers in New Zealand are at risk of leptospirosis, suggesting that the pathogen is still present, despite widespread vaccination programs in dairy cattle against leptospirosis ${ }^{15,16}$.

Between 1979 and 1981 there were 208 confirmed cases of leptospirosis in humans in Victoria. These included 101 farmers, 44 meat workers, and 11 meat inspectors ${ }^{26}$. Occupations were not stated for 35 individuals and, of the remaining 17, eight had occupational contact with animals, but details of the species involved were not recorded. In 2010, 14 cases of leptospirosis were officially notified in Victoria, 10 of which were work associated. Six dairy farmers, two farm hands and two beef cattle farmers were affected ${ }^{27}$. It is not clear whether the decrease in the number of occupational leptospirosis diagnoses in humans in Victoria $^{28}$ is due to a decrease in the incidence of leptospirosis in cattle (possibly associated with an increase in the number of cattle vaccinated for disease) or failure of the medical community to diagnose cases of disease (due to the similarity of the symptoms of leptospirosis with those of other diseases).

Over recent years more human cases of leptospirosis have been reported in both Queensland and NSW. In 2018 a study in NSW reported an outbreak among workers in a raspberry orchard in which rodents were implicated as the source of infection ${ }^{29}$. A retrospective study in an intensive care hospital in tropical Queensland has reported confirmed cases between 1998 and 2018 without information on the source of the infection ${ }^{30}$. 
Despite the smaller number of human cases of leptospirosis in Victoria, data obtained from the Victorian Department of Health and Human Services at the end of 2018, showed that $45 \%$ of all confirmed human leptospirosis cases from January 2016 to September 2018 were still likely to be associated with farming or farm animals (Dept Health and Human Services, Victoria pers comm, 2019). Out of all human cases reported L. Hardjo was still the predominant pathogen, and the majority of L. Hardjo positive cases were associated with farming and/or farm animals.

The aim of the current study was to estimate the prevalence of leptospirosis in dairy herds in South-Western Victoria, Australia. A secondary aim was to estimate the proportion of dairy herds using leptospirosis vaccination as a routine management tool and the proportion of herds using vaccination according to all of the manufacturer's recommendations.

\section{Material and methods}

Ethics approval for this study was provided by the University of Melbourne Human Research Ethics Committee (Ethics ID: 1647214.1) and the Faculty of Veterinary and Agriculture Sciences animal ethics committee (Ethics ID: 1613950.1).

The source population for this study was the $4126^{6}$ dairy herds in South-Western Victoria, Australia that sold milk for human consumption in 2016. The eligible population were 220 herds from the source population whose herd manager were clients of a large veterinary practice in Warrnambool, located in the centre of the study area (Figure 1). A second criteria 
for eligibility was that individual cows within herds had to be pregnancy tested following the most recent mating period. The study group comprised those members of the eligible population whose herd managers agreed to take part in the study following an invitation distributed via a practice newsletter in October $2016(n=60)$. Initial attempts to identifying a similar number of vaccinated and unvaccinated herds for sampling was unsuccessful as it is uncommon for clients of the practice not to use a leptospirosis vaccine.

Between November 2016 and August 2017 each herd enrolled in the study was visited by the first author. At the time of each herd visit a questionnaire was provided to the herd owner or herd manager. The questionnaire explored whether leptospirosis vaccination was routinely used and, if vaccination was used whether it was used in a way that was consistent with all of the manufacturer's recommendations. Additional details collected included the average size of the milking herd, the numbers of animals in each age class (calves, heifers and adult milking cows), the predominant breed of animals in the milking herd, the size of the effective milking area, details of the presence of other domestic animals resident on the property, presence or absence of biosecurity practices and whether or not cases of leptospirosis had been diagnosed in workers or owner-managers of the property in the past.

Sample size calculations were carried out to determine the appropriate number of cows to sample per herd to detect at least one cow with leptospirosis. On the basis of client records, we calculated required sample sizes assuming that the typical number of milking cows in dairy herds in South-Western Victoria ranged from 100 to 1500 cows. We designed the study 
to detect herds that had a within-herd prevalence of leptospirosis of $20 \%$ as it was thought this level would likely be biologically important in terms of the possibility of zoonotic infection risk. Using a test with a diagnostic sensitivity of $95 \%$, to be $95 \%$ confident that at least one cow would return a positive test if the within-herd prevalence of leptospirosis was $20 \%$, at least 15 cows were needed to be sampled and tested from each herd ${ }^{31,32}$.

Studies in the United Kingdom ${ }^{17}$ demonstrated a correlation between delayed calving to conception intervals and the presence of leptospirosis. Reduced conception rates were correlated with leptospira titres and impaired fertility was observed in the year in which infection was identified. Thus, to increase the probability of detecting leptospira-positive cows if they were present, we asked herd managers to present for sampling 15 cows that had either failed to become pregnant during the mating period or had taken longer to conceive, compared with the rest of the herd. Individual cows were chosen opportunistically by the herd manager based on the criteria provided.

Samples were collected shortly after pregnancy testing had finished, to identify non-pregnant cows. Herds with less than 15 non-pregnant cows (of which there were four) were asked to present cows that had delayed conception (conceived at the latest date within the mating period).

At the time of each herd visit cows selected for sampling were injected with furosemide (Frusemide Injection, Ilium Veterinary Products, New South Wales, Australia). This has been 
shown to change the osmolarity of the urine and to increase the likelihood of harvesting leptospira if they are present in the renal tubular lumen ${ }^{23}$. Urine was collected from the first and second voiding post administration of furosemide, but only the second voided sample was used for analysis, based on findings of Nervig and Garrett (1979) ${ }^{33}$. Following collection, urine samples were transported in a cool box with ice blocks to the laboratory within 24 hours. Once received at the laboratory $1 \mathrm{~mL}$ of urine per sample was centrifuged at $14,000 \mathrm{~g}$ to pelletise any cellular tissue. For the pooled samples $1 \mathrm{~mL}$ of urine from each cow tested per herd was filter centrifuged using a Pall Macrosep $0.2 \mu \mathrm{m}$ centrifugal filter. Then $60 \mu \mathrm{L}$ of PBS was added to the pellet before the sample was either run for DNA extraction or frozen to be run at a later stage for DNA extraction. Freezing and thawing of urine samples without preparation was less successful, thus cell pellets from samples arriving at the lab had to be prepared on the day of arrival. For extraction of the DNA, Qiagen DNeasy extraction was used.

\section{Leptospira PCR}

DNA extraction and PCR tests were undertaken at a commercial laboratory (Ace Laboratory Services, East Bendigo, 12 Gildea Lane, Victoria 3550) using primers and probes described in Table 1 (Zoetis Australia, Rhodes, New South Wales 2138).

During DNA extraction it was noted that some leptospira were caught in the Macrosep filter, hence the filter was swabbed firmly with a PBS wetted swab. DNA on the swab was also extracted via DNeasy and tested as a replicate to the concentrated urines for confirmation. 
A positive control and a negative control were added to each PCR run to confirm specific and efficient amplification. Samples were considered positive if their cyclic threshold (Ct) value was 36 or less. Samples with Ct value greater than 36 were interpreted as either weak positive or inconclusive. Samples were deemed to be negative if no leptospira were detected after 40 cycles.

Samples were first tested at both the pooled (1mL from each individual sample) and individual cow level for Leptospira spp. Positive herds were then tested with serovar specific PCR for L. borgpetersenii sv. Hardjo and L. interrogans sv. Pomona.

\section{Statistical methods}

We report the frequency of leptospira-positive herds as prevalence, expressed as the number of leptospira-positive herds per 100 herds at risk. The frequency of herd managers not vaccinating their herds according to label instructions is reported as a prevalence in the same manner. Exact confidence intervals for each prevalence estimate were calculated using the methods described by Collett $(1979)^{34}$. Unconditional associations between each of the binary herd-level characteristics from the questionnaire and herd leptospirosis status were quantified using the prevalence ratio with the Haldane-Anscombe correction ${ }^{35,36}$ used for comparisons where the frequency of any cell of the $2 \times 2$ table was equal to zero. Prevalence ratios were computed using the contributed epiR package in R (R Core team, 2018). 


\section{Results}

Initially 60 herd managers agreed to take part in the study. Seven of these herd managers subsequently withdrew due to time restrictions or an inability to agree on a mutually convenient time for the herd visit.

Of the remaining 53 herds that participated in the study 32 herds were seasonal calving herds, 21 had split-calving patterns and one herd was identified as being a year-round calving herd. Most herds that vaccinated their adult cows had done so at least 3 months prior to the study (85\%), with the majority having vaccinated their cows at least seven months prior to the study (58\%).

Data regarding the age of 724 cows from 795 participating cows was collected. On this basis cows were categorised into 3 groups: young ( 2 to 4 years of age; $n=258$ ), middle-age ( 5 to 7 years of age; $n=297$ ) and older cows (over the age of 8 years old; $n=169$ ). No age was provided for 71 cows.

Of the 53 herds, only one was identified as being fully unvaccinated against leptospirosis. The most commonly used vaccines within the study were Ultravac ${ }^{\circledR} 7$ in 1 (Zoetis, Australia) and Websters ${ }^{\circledR} 7$ in 1 vaccine for cattle (Virbac, Australia). Either 7in1 vaccine covers for L. interrogans sv Pomona, L. borgpetersenii sv Hardjo and 5 clostridial diseases. None of the participant herd managers used monovalent vaccines. Only 16 herd managers vaccinated their herds in complete accordance with the manufacturer's label instructions (i.e. calves were 
vaccinated within the first 3 months of life; the time between the initial two vaccines was 4-6 weeks; and calves that received their second injection at less than 12 weeks of age received a third vaccination up to 6 months later before going on to receive yearly boosters).

Managers of 19 herds vaccinated all their stock (all age groups) but, for at least one part of the program, did not vaccinate at time intervals that were in accordance with the product label. Examples of non-compliance with label directions included: prolonged interval between the first two vaccinations; failure to administer the 6-month booster when calves received their initial two vaccinations at less than 12 weeks of age; calves being over 3 months of age at their initial vaccination; and not all cows receiving their 12-monthly booster vaccination. Herd managers of 17 herds stated that they vaccinated part of their herds, such as calves or calves and youngstock only.

Thirty-five of the 52 herd managers that vaccinated their herds were non-compliant in at least one aspect of the manufacturer's label instructions. There were 67 (95\% CI 54 to 78) noncompliant vaccinated herds per 100 vaccinated herds at risk.

Unconditional associations between each of the binary herd-level characteristics and herd leptospirosis status are shown in Table 2. Of the 11 herd-level characteristics that were assessed, only the presence of a previous diagnosis of leptospirosis in a human resident on the farm was statistically significantly associated with the probability of a herd being leptospirosis positive at the time of testing. For herds where a case of leptospirosis had been 
diagnosed in humans in the past, the risk of being leptospirosis positive was 12.9 (95\% CI 1.5 to 111) times more than herds were no cases of human leptospirosis had been diagnosed.

Three of the 53 herds that took part in the study were identified as being infected with leptospira. One of the 3 herds had three positive samples, the other two each had five positive and one inconclusive sample. Individual-sample testing of the remaining 50 herd samples identified an additional positive herd with one positive and one inconclusive sample. This gave a total of four positive herds out of the 53 herds that participated in the study, a prevalence of 8 (95\% CI 1 to 13) leptospirosis-positive herds per 100 herds at risk. Once individual-cow positive samples were identified, serovar specific PCRs were run for Leptospira borgpetersenii serovar Hardjo and Leptospira interrogans serovar Pomona. The number of cow tests that were positive, inconclusive and negative to Leptospirosis serovars based on PCR is shown in Table 3. Of 17 positive or inconclusive individual urine samples, eight samples were positive and four were weak positive or inconclusive for Leptospira borgpetersenii serovar Hardjo. No positive samples were identified as positive for Leptospira interrogans serovar Pomona. Five samples were negative for both serovars.

Of the 17 cows which were positive or inconclusive for leptospires 13 were young animals, two were middle-aged and only one was above 8 years of age. Seventy one percent of the leptospira positive individual urine samples were associated with $L$. borgpetersenii serovar Hardjo, containing both positive and inconclusive results. 


\section{Discussion}

It is possible that our study population was biased toward herd managers that vaccinate for leptospirosis because those who do not vaccinate might have been less likely to respond to the study invitation. If this was the case, our study is likely to underestimate the true prevalence of leptospirosis-positive dairy herds in South-West Victoria.

\section{Survey}

The survey focused on trying to elicit the correct information regarding vaccination regimes by asking the same question in three different ways. Initially the herd managers were asked whether they vaccinated their herds. This was followed in more detail by asking if they vaccinated calves, young-stock, heifers, cows and bulls. Thereafter, questions went into further detail by asking when and at what age each group was vaccinated. In this way we attempted to identify issues with the vaccination program whilst attempting to confirm whether answers had been given accurately.

Ignoring the single unvaccinated herd, enrolled herds could be categorised into three similar sized groups: compliant to label-use vaccinated herds (all age groups), non-compliant vaccinated herds (all age groups) and partly-vaccinated herds. As the partly vaccinated herds were also non-compliant vaccinated herds, these groups were merged for subsequent analysis. 
Many herd managers and their workers believed that they were vaccinating correctly, even when they did not comply fully with vaccine label directions. This may be partly because most vaccinate their stock against several different diseases using vaccines with different regimes and it is easy to make mistakes or get confused. Some managers were aware of the incorrect timing of their vaccination regime and were curious to see whether this would be reflected in their sample results. An example of a reason for some cattle not to be vaccinated was that on some farms non-pregnant cows did not receive an annual booster as herd managers would only vaccinate the herd at drying-off. Non-pregnant cows that are carried over to the next season are often not dried off.

The number of herd managers that identified either themselves, one of their family members or one of their staff as having had leptospirosis in the past 25 years is an important finding. Most herd managers in this group vaccinated their herds for leptospirosis, probably due to their own experience with the debilitating impact that clinical leptospirosis can have on human health. We expected that leptospirosis control programs would be more effectively applied in those herds where there had been previous human cases of leptospirosis, but this was found not to be the case. It is of concern that, despite the relatively low numbers of herds in our study, there was still a significant positive association between the presence of human disease and the herd being positive for leptospira.

Urine testing

This article is protected by copyright. All rights reserved. 
Where vaccinating for leptospirosis is not common practice, serological studies may be reflective of exposure to the bacterium. However, serological studies are less appropriate where vaccination of dairy herds is a relatively common practice. The development of 7-in-1 vaccines (5 clostridial diseases and 2 leptospira serovars) in Australia has led to leptospirosis vaccination being a common routine management procedure because herd managers are familiar with the need to vaccinate for clostridial diseases and there is no extra work involved in also vaccinating for leptospirosis.

Previous work suggests that microscopic agglutination test (MAT) results after vaccination are weak and of shorter duration, whereas titres to natural infection are stronger and persist for longer ${ }^{37}$. It is difficult to use a MAT result to distinguish between vaccinated and naturally exposed cattle because vaccinated cattle that are subsequently exposed to the live organism can have very strong antibody responses despite being protected. Testing urine for the presence of leptospires is a much more reliable way to identify an infection ${ }^{38}$ although shedding of leptospirosis in cows can be intermittent, continuous for a period, or a combination of the two, meaning that this approach will likely underestimate the true proportion of infected cows.

A study investigating the efficacy of an Australian bivalent leptospira vaccine for cattle showed that animals that are vaccinated correctly and at a young age, before being exposed to the bacterium, did not shed the pathogen ${ }^{39}$. However, this has not been demonstrated for all leptospirosis vaccines on the market. Preventing shedding of the bacterium should be the 
main aim of vaccination, as this helps to minimise the distribution of the disease to both animals and humans.

\section{Vaccination label directions}

Young animals are more typically naïve to the bacteria as they have not had as much time in which to encounter an infection through transfer of bacteria from infected older stock. In addition, the organism can be shed over a period of up to 60 weeks ${ }^{20}$. Thus, the risk of exposure of calves is ongoing. It is important when implementing a vaccination program that cattle are protected over their lifetime to ensure that there are no 'windows of opportunity' for infection, hence the importance of compliance with vaccine label directions.

In this study we identified animals of any age with suboptimal reproductive performance going through the milking shed. This meant that both first time calving cows and older animals were tested. Older animals could be at risk of infection especially in herds where only calves and young-stock were vaccinated, considering that a long-term efficacy study of commercial leptospira vaccine showed good protection of up to 55 weeks ${ }^{4}$. At 55 weeks only $2.7 \%$ of vaccinated heifers were found with leptospiruria compared with $58.5 \%$ of unvaccinated heifers (although it should be noted that this study used a leptospira only vaccine, not the product in common use in our study).

Previous studies identified a correlation between reproductive problems and seropositive cows, although these studies also identified a higher fertility drop within the year of diagnosis 
of leptospira , and that fertility recovered slowly after the introduction ${ }^{17,18}$. Further studies have identified leptospira in the reproductive tract of cattle, which might be another reason fertility in cows with leptospirosis could be decreased ${ }^{40-42}$. Despite the five studies cited here being from overseas, we preferentially selected cows with fertility issues for sampling.

As mentioned previously, L. borgpetersenii sv Hardjo has not been shown to be a major cause of abortions ${ }^{12}$ and little information on the association between Hardjo infection and fertility has been reported in Australia. It appears that L. borgpetersenii sv Hardjo is less pathogenic than L. interrogans sv Hardjo (subtype Hardjoprajitno), which could be the reason abortions and fertility issues are more often described in UK dairies than in Australia ${ }^{43}$.

Furosemide was used to induce urination but also to decrease the osmolarity of the urine which made the urine more favourable for survival of leptospira ${ }^{44}$. Initial voiding typically took around 10-15 minutes in this trial (range 5 - 30 minutes) and the average time between first and second voiding was 17 minutes. First voided urine was light to dark yellow possibly reflecting the cow's hydration status and ambient temperature on the day of collection. Second voiding produced clear urine in all cases regardless of the colour of the urine from the first voiding.

Leptospira have a low survival time in urine, especially in more acidic urine as the bacterium prefers neutral to alkaline urine which would be the case in healthy cattle. The survival of leptospira in urine samples can also be affected by sunlight, temperature and dilution of the 
sample. A study performed in Malaysia identified leptospirosis survival of 2-48 hours in undiluted urine at $4^{\circ}$ Celcius ${ }^{45}$. Samples in our study were cooled and transported to the lab within 24 hours whenever possible. It is possible that leptospira might have died and lysed during the transport to the lab, and DNA could therefore be lost with the supernatant when being centrifuged ${ }^{46}$.

The testing method chosen for this study was qPCR, as PCR testing is very sensitive and specific and relatively easy to perform. Although PCR will not distinguish between live or dead bacteria, this might be helpful if leptospira die during the transport and preparation process of the samples, as it will still identify leptospira positive samples.

Whilst our study has some limitations associated with sample size, the opportunistic selection of cows by the herd managers and the diagnostic sensitivity of the tests used, these limitations are likely to mean our estimates of the herd prevalence of leptospirosis to be 10 infected herds per 100 at risk are conservative. Leptospirosis is an important zoonotic disease and further work is warranted to understand why such a relatively high prevalence of leptospirosis exists amongst dairy farms, despite the implementation of leptospirosis vaccination over many years. Ineffective vaccination due to incorrect storage of vaccine (temperature, duration) and incorrect administration of vaccine by herd managers, which have been reported as reasons for failure of veterinary vaccines, may be factors. It is also possible that poor compliance with vaccine label directions, as reported here, is a contributing factor. 


\section{Conclusions}

Leptospira are present in South-Western Victorian dairy herds and remain a risk to herd managers, their families and employees. We estimate that almost ten percent of dairy herds are leptospirosis positive, despite vaccination programs being implemented on most farms. Lack of compliance with vaccine label directions is one reason why the prevalence of leptospirosis remains relatively high. We conclude that further education of herd managers and their employees is needed to reiterate the risks and consequences of infection and deliver clear messages around the importance of good vaccine label compliance.

\section{Acknowledgements}

Funding for this project came from Zoetis Australia. A special thanks to Sally Oswin from Zoetis, who helped organising the project and for her constant support. The authors would also like to thank Dairy Australia for sponsoring the dairy cattle residency program which enabled this project, the veterinary clinic for their support and the herd managers involved.

\section{Conflict of interest}

The authors declare no conflict of interest.

\section{References}

1. Adler B. Leptospirosis and Leptospira. Adler B, editor. Curr Top Microbiol Immunol Springer, 2014;387.

This article is protected by copyright. All rights reserved. 
2. Milner AR, Wilks CR, Calvert K. The prevalence of antibodies to members of Leptospira interrogans in cattle. Aust Vet J 1980;56:327-330.

3. Sutherland A. Surveys on human leptospirosis. AVA News 1988;1-2.

4. Hancock GA, Wilks CR, Kotiw M et al. The long term efficacy of a Hardjo-pomona vaccine in preventing leptospiruria in cattle exposed to natural challenge with Leptospira interrogans serovar hardjo. Aust Vet J 1984;61:54-56.

5. Bolin CA, Alt DP. Use of a monovalent leptospiral vaccine to prevent renal colonization and urinary shedding in cattle exposed to Leptospira borgpetersenii serovar hardjo. Am J Vet Res 2001;62:995-1000.

6. Dibden J, Cocklin C. Re-mapping regulatory space: The new governance of Australian dairying. Geoforum 2010;41:410-422. http://dx.doi.org/10.1016/j.geoforum.2009.11.007.

7. Beggs DS, Fisher AD, Jongman EC et al. A survey of Australian dairy farmers to investigate animal welfare risks associated with increasing scale of production. J Dairy Sci 2015;98:5330-5338.

8. Dairy Australia. Dairy Situation and Outlook June 2018. 2018.

9. Jarvis J, Peel V. Tourists for hire: International working holidaymakers in a work based destination in regional Australia. Tour Manag 2013;37:114-124. http://dx.doi.org/10.1016/j.tourman.2012.10.014.

10. Waitkins SA. Leptospirosis as an occupational disease. Occup Environ Med 2008;43:721-725.

11. Gordon LM, Sci BA. Leptospira interrogans Serotype Hardjo outbreak in a Victorian 
Dairy Herd and Associated Infection in Man. Aust Vet J 1977;53:227-229.

12. Chappel RJ, Millar BD, Adler B et al. Leptospira interrogans serovar hardjo is not a major cause of bovine abortion in Victoria. Aust Vet $J$ 1989;66:330-333.

13. Peterson JE. Leptospirosis of cattle and pigs in western Australia. Aust Vet J $1951 ; 27: 40-43$.

14. Carroll AG, Campbell RS. Reproductive and leptospiral studies on beef cattle in central Queensland. Aust Vet J 1987;64:1-5.

15. Sanhueza JM, Baker MG, Benschop J et al. Estimation of the burden of leptospirosis in New Zealand. Zoonoses Public Health 2020;67:167-176.

16. Yupiana Y, Vallee E, Wilson P et al. Emerging Leptospira strain poses public health risk for dairy farmers in New Zealand. Prev Vet Med 2019;170:104727. https://doi.org/10.1016/j.prevetmed.2019.104727.

17. Dhaliwal G., Murray RD, Dobson H et al. Reduced conception rates in dairy cattle associated with serological evidence of Leptospirosis interrogans serovar hardjo infection. Vet Rec 1996;139:110-114.

18. Dhaliwal GS, Murray RD, Ellis WA. Reproductive performance of dairy herds infected with Leptospira interrogans serovar hardjo relative to the year of diagnosis. Vet Rec 1996;138:272-277.

19. Grooms DL. Reproductive losses caused by bovine viral diarrhea virus and leptospirosis. Theriogenology 2006;66:624-628.

20. Restriction endonuclease analysis of Australian isolates of Leptospira interrogans serovar hardjo. Aust Vet J 1989;66:183-184.

This article is protected by copyright. All rights reserved. 
21. Levett PN. Leptospirosis Leptospirosis. Clin Microbiol Rev 2001;14:296-326.

22. $\quad$ Lau CL, Townell N, Stephenson E et al. Leptospirosis. R Aust J Gen Pract 2018;47.

23. Doerfler W, Böhm P. The Molecular Repertoire of Adenoviruses I Virion Structure and Infection. Springer 1995. http://www.springer.com/us/book/9783662450581.

24. Rodriguez-gonzalez I, Fillonneau C, Blanchet B et al. par la protection passive de rongeurs de laboratoire Efficacy of the Spirolept ${ }^{\circledR}$ vaccine against human leptospirosis estimated by passive sera-protection of laboratory rodents. Med Mal Infect 2004;34:196-200.

25. Verma R, Khanna P, Chawla S. Whole-cell inactivated Leptospirosis vaccine. Landes Biosci Hum Vaccines Immunother 2013;763-765.

26. Swart KS, Wilks CR, Jackson KB et al. Human leptospirosis in Victoria. Med J Aust $1983 ; 1: 460-463$.

27. Department of Health. Surveillance of notifiable infectious diseases in Victoria,2010. 2012:73-75. http://ideas.health.vic.gov.au/surveillance/annual-reports.asp. Retrieved May 92020.

28. Australian Government Department of Health. Human cases of Leptospirosis in Australia. 2018. http://www9.health.gov.au/cda/source/cda-index.cfm. Retrieved May 92018.

29. Katelaris AL, Glasgow K, Lawrence K et al. Investigation and response to an outbreak of leptospirosis among raspberry workers in Australia, 2018. Zoonoses Public Health 2020;67:35-43.

30. Smith S, Liu YH, Carter A et al. Severe leptospirosis in tropical Australia: Optimising 
intensive care unit management to reduce mortality. PLoS Negl Trop Dis 2019;13:e0007929.

31. Dohoo I, Martin S, Stryhn S. Veterinary epidemiologic research. AVC Inc, Charlottetown, 2009.

32. Cannon RM. Sense and sensitivity - Designing surveys based on an imperfect test. Prev Vet Med 2001;49:141-163.

33. Nervig RM, Garrett LA. Use of furosemide to obtain bovine urine samples for leptospiral isolation. Am J Vet Res 1979;40:1197-1200.

34. Bocca Raton F, Chapman, Hall/CRC. Modelling binary data. 1999. https://www.rproject.org/.

35. Haldane AJBS. Biometrika Trust The Mean and Variance of $\mid$ chi2, When Used as a Test of Homogeneity, When Expectations are Small. Oxford Jourrnals 1940;31:346355. https://www.jstor.org/stable/2332614.

36. Anscombe F. On estimating binomial response relations. Biometrika 1956;43:461464.

37. Marshall RB, Broughton ES, Hellstrom JS. Protection of cadle against natural challenge with leptospira interrogans serovar hardjo using a hardjo-pomona vaccine. $N$ Z Vet J 1979;27:114-116.

38. Hamond C, Martins G, Loureiro AP et al. Urinary PCR as an increasingly useful tool for an accurate diagnosis of leptospirosis in livestock. Vet Res Commun 2014;38:8185.

39. Alexander AM, Booth DH, Bouma A et al. Efficacy of an Australian Bivalent 
Leptospira Vaccine for Cattle. Aust Jounal Biotechnol 1989;3:143-146.

40. Ellis WA, Songer JG, Montgomery J et al. Prevalence of Leptospira interrogans serovar hardjo in the genital and urinary tracts of non-pregnant cattle. Vet Rec 1986;118:11-13.

41. Cabral Pires B, Berzin Grapiglia J, Moreira L et al. Occurrence of uterine carriers for Leptospira interrogans on slaughtered cows. Microb Pathog 2018;114:163-165.

42. Bielanski A, Surujballi O, Thomas EG et al. Sanitary status of oocytes and embryos collected from heifers experimentally exposed to Leptospira borgpetersenii serovar hardjobovis. Anim Reprod Sci 1998;54:65-73.

43. Smith CR, Ketterer PJ, McGowan MR et al. A review of laboratory techniques and their use in the diagnosis of Leptospira interrogans serovar hardjo infection in cattle. Aust Vet J 1994;71:290-294.

44. Nervig RM, Garrett LA. Use of furosemide to obtain bovine urine samples for leptospiral isolation. Am J Vet Res 1979;40:1197-1200.

45. Khairani-Bejo S. et al. The Survival of Leptospira interrogans Serovar Hardjo in the Malaysian Environment.Pdf. J Anim Veterinay Adv 2004;3:123-129.

46. Lucchesi PMA, Arroyo GH, Etcheverría AI et al. Recommendations for the detection of Leptospira in urine by PCR. Rev Soc Bras Med Trop 2004;37:131-134.

This article is protected by copyright. All rights reserved. 


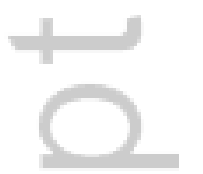

4 
Table 1: Primers and probes used in Leptospira PCR (Zoetis Australia, Rhodes, New South Wales 2138)

\begin{tabular}{|c|c|c|}
\hline Oligo & Sequence & Dye \\
\hline \multicolumn{3}{|c|}{ Pomona specific } \\
\hline P7 & 5'- TTACAGACGGGAGAGATACAC-3' & \\
\hline P8 & 5'- AAGGAATACGATCAGGAGTITG-3' & \\
\hline Pomona Probe & 5'- TGCATCGAGATTATTTCCTTCTTGG -3' & FAM-IBFQ \\
\hline \multicolumn{3}{|c|}{ Hardjo specific } \\
\hline $\mathrm{H} 11$ & 5'- CCATTGTTCCTGAAGTTGATG-3' & \\
\hline $\mathrm{H} 12$ & 5'- CGATTCCTGTGGCAAAGC-3' & \\
\hline Hardjo Probe & 5'- TTCGAACACAAGGATTACGCTाT -3' & FAM-IBFQ \\
\hline \multicolumn{3}{|l|}{ Lepto all spp } \\
\hline LipL32-45F & 5'-AAG CAT TAC CGC TTG TGG TG-3' & \\
\hline LipL32-286R & $5^{\prime}$-GAA CTC CCA ПTा CAG CGA $\Pi 1-3^{\prime}$ & \\
\hline LipL32-Pr & 5'-AA AGC CAG GAC AAG CGC CG-3' & FAM-IBFQ \\
\hline
\end{tabular}

This article is protected by copyright. All rights reserved. 
Table 2: The prevalence of and risk factors for leptospirosis in South-Western Victorian dairy herds, 2017. Unconditional associations between herd leptospirosis status and eleven explanatory variables.

\begin{tabular}{|c|c|c|c|}
\hline \multirow[t]{2}{*}{ Herd-level characteristic } & \multicolumn{2}{|c|}{ Herd leptospirosis status } & \multirow[t]{2}{*}{ PR $(95 \% \mathrm{Cl})^{a}$} \\
\hline & Positive & Negative & \\
\hline \multicolumn{4}{|l|}{ Vaccination of calves: } \\
\hline No & 0 & 4 & $1.7(0.23,12)^{b}$ \\
\hline Yes & 4 & 44 & Reference \\
\hline \multicolumn{4}{|l|}{ Vaccination of young stock: } \\
\hline No & 2 & 11 & $3.0(0.47,19)$ \\
\hline Yes & 2 & 37 & Reference \\
\hline \multicolumn{4}{|l|}{ Vaccination of adult cattle: } \\
\hline No & 2 & 15 & $2.1(0.32,13)$ \\
\hline Yes & 2 & 33 & Reference \\
\hline \multicolumn{4}{|l|}{ Vaccination of entire herd: } \\
\hline Non-compliant & 4 & 32 & $2.4(0.30,19)^{b}$ \\
\hline Compliant & 0 & 16 & Reference \\
\hline \multicolumn{4}{|l|}{ Timing of vaccination: } \\
\hline Non-compliant & 3 & 22 & $3.2(0.36,29)$ \\
\hline Compliant & 1 & 26 & Reference \\
\hline \multicolumn{4}{|l|}{ Heifers reared of farm: } \\
\hline Yes & 3 & 34 & $1.3(0.15,11)$ \\
\hline No & 1 & 15 & Reference \\
\hline \multicolumn{4}{|c|}{ Other animals present on farm: } \\
\hline Yes & 2 & 31 & $0.6(0.09,4.0)$ \\
\hline No & 2 & 18 & Reference \\
\hline \multicolumn{4}{|l|}{ Pigs present on farm: } \\
\hline Yes & 0 & 7 & $1.1(0.14,8.1)^{b}$ \\
\hline No & 4 & 42 & Reference \\
\hline \multicolumn{4}{|l|}{ Open herd: } \\
\hline Yes & 2 & 16 & $1.9(0.30,13)$ \\
\hline No & 2 & 33 & Reference \\
\hline \multicolumn{4}{|l|}{ Cattle quarantined: } \\
\hline Yes & 0 & 7 & $1.0(0.13,7.6)^{b}$ \\
\hline No & 4 & 39 & Reference \\
\hline \multicolumn{4}{|l|}{ Human cases of leptospirosis } \\
\hline Yes & 3 & 7 & $13(1.5,110)$ \\
\hline No & 1 & 42 & Reference \\
\hline
\end{tabular}

${ }^{\mathrm{a}}$ PR: prevalence ratio; $\mathrm{CI}$ : confidence interval.

${ }^{\mathrm{b}}$ Haldane-Anscombe corrected prevalence ratio 
Table 3: The prevalence of and risk factors for leptospirosis in South-Western Victorian dairy herds, 2017. Counts of cows positive, inconclusive and negative to Leptospirosis serovars based on PCR.

\begin{tabular}{lllll}
\hline Isolate & Positive & $\begin{array}{c}\text { Cow status } \\
\text { Inconclusive }\end{array}$ & Negative & \\
\hline L. hardjo & 8 & 4 & 5 & $71(44 \text { to } 90)^{\mathrm{b}}$ \\
L. pomona & 0 & 0 & 17 & $0(0$ to 20$)$ \\
\hline
\end{tabular}

${ }^{\mathrm{a}}$ CI: confidence interval.

b. Includes Leptospira positive and inconclusive isolates. 
Figure 1: Map of South-Western Victoria, Australia showing the location of study farms. Locations have been jittered by 1 kilometre to preserve anonymity.

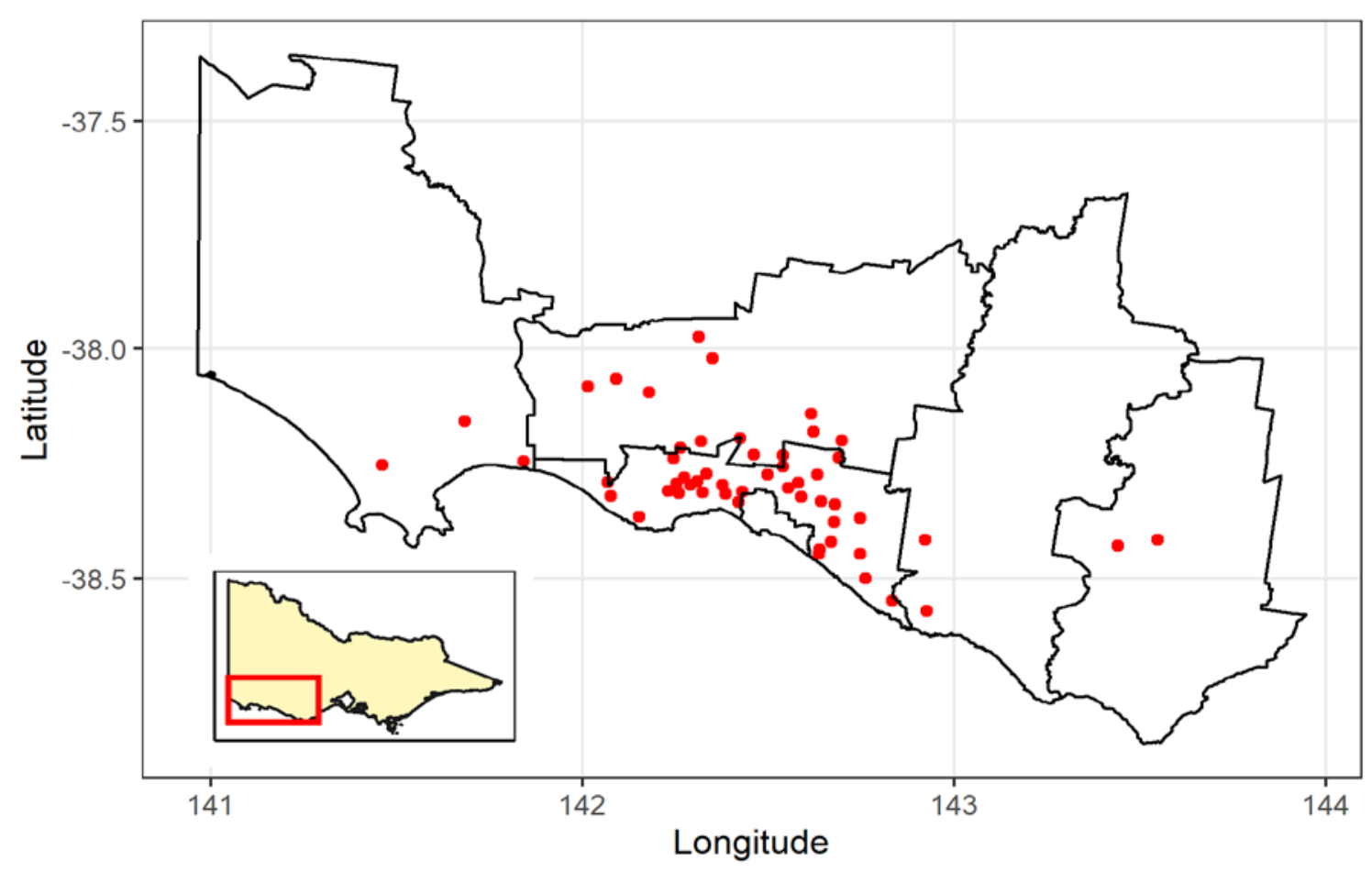

This article is protected by copyright. All rights reserved. 


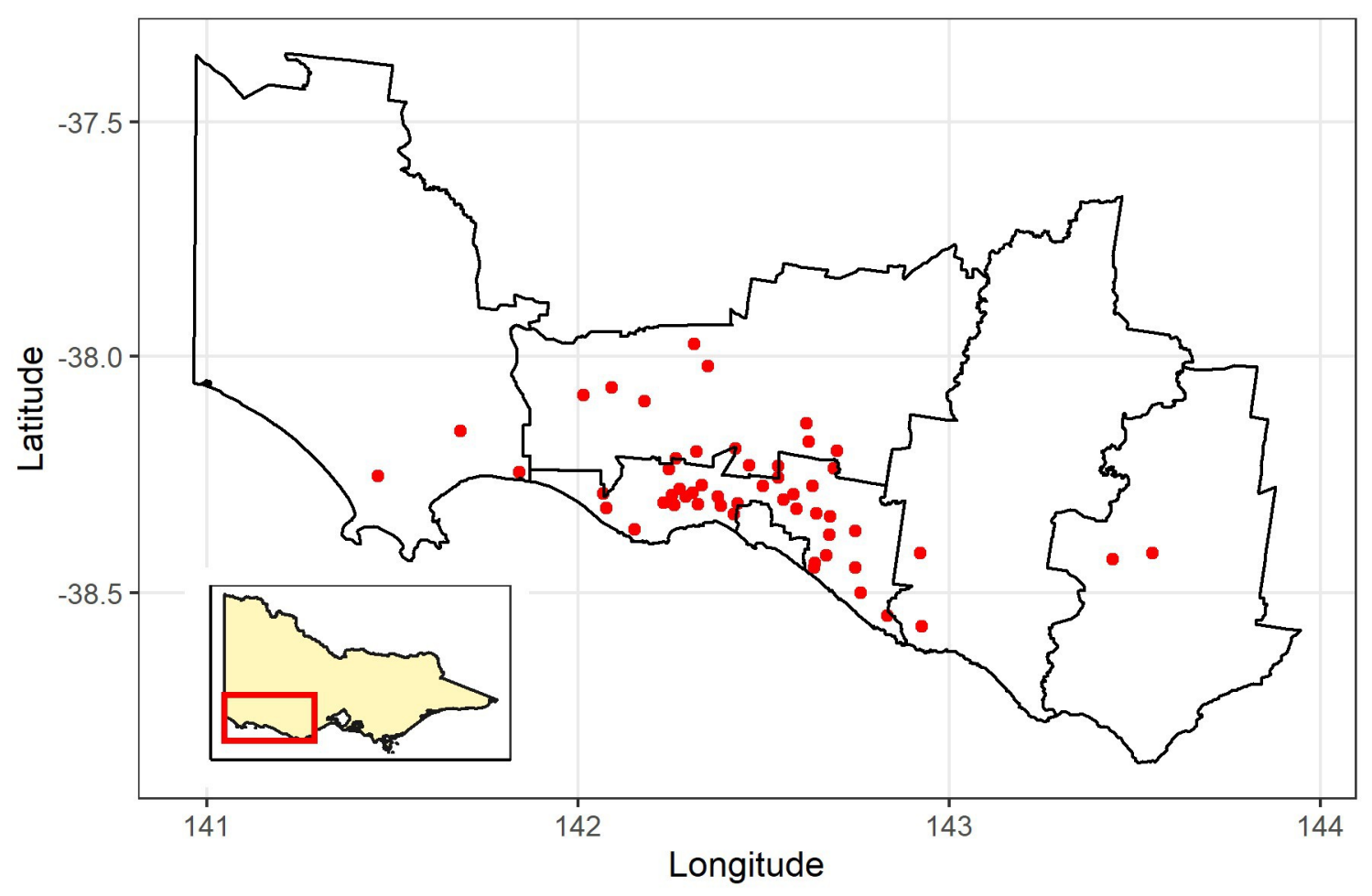

AVJ_12984_Figure 1 - Location of study farms.jpg

This article is protected by copyright. All rights reserved. 


\section{University Library}

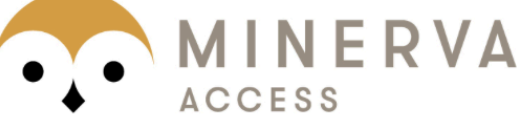

A gateway to Melbourne's research publications

Minerva Access is the Institutional Repository of The University of Melbourne

Author/s:

Erregger, E;Stevenson, MA;Beggs, DS;Oswin, S;Jagoe, SP;Mansell, PD;Pyman, MF

Title:

A cross-sectional pilot study to estimate the prevalence of and risk factors for leptospirosis inSouth-WesternVictorian dairy herds, 2017

Date:

2020-07-20

Citation:

Erregger, E., Stevenson, M. A., Beggs, D. S., Oswin, S., Jagoe, S. P., Mansell, P. D. \& Pyman, M. F. (2020). A cross-sectional pilot study to estimate the prevalence of and risk factors for leptospirosis inSouth-WesternVictorian dairy herds, 2017. AUSTRALIAN VETERINARY JOURNAL, 98 (9), pp.417-423. https://doi.org/10.1111/avj.12984.

Persistent Link:

http://hdl.handle.net/11343/276028 